\section{Relation between Bull Sperm Respiratory Burst Activity and the In Vitro Fertilization Rate: A New Approach to Evaluate Bull's Fertility}

\author{
António Chaveiro, ${ }^{1,2}$ Sofia Mota de Azevedo, ${ }^{1}$ Pedro Santos, ${ }^{1}$ Isabel Carvalhais,,${ }^{1,2}$ \\ Marwa Faheem, ${ }^{1,2}$ Afrooz Habibi, ${ }^{1,2}$ and Fernando Moreira da Silva ${ }^{1,2}$ \\ ${ }^{1}$ Animal Reproduction, Department of Agrarian Sciences, University of the Azores, 9701-851 Angra do Heroísmo, Portugal \\ ${ }^{2}$ CITA-A, University of the Azores, 9701-851 Angra do Heroísmo, Portugal
}

Correspondence should be addressed to Fernando Moreira da Silva, jsilva@uac.pt

Received 30 July 2009; Accepted 7 September 2009

Copyright ( 2010 António Chaveiro et al. This is an open access article distributed under the Creative Commons Attribution License, which permits unrestricted use, distribution, and reproduction in any medium, provided the original work is properly cited.

Sperm of 8 different bulls ( 3 ejaculates per bull) was used in the present study to evaluate the oxidative burst activity of each individual sperm cell by flow cytometry, correlating this data with the results of in vitro fertilization as well as the further embryo development to the stage of blastocyst. After thawing, the straw content was split in two identical parts. One was employed for the in vitro fertilization, while the other was used for flow cytometry to evaluate sperm oxidative burst activity by an assay using $2^{\prime}, 7^{\prime}$-dichlorofluorescin diacetate, which based on the oxidation of nonfluorescent DCFH-DA to highly fluorescent $2^{\prime}, 7^{\prime}-$ dichlorofluorescein (DCF) intracellularly during the respiratory burst. As far as embryo production and the sperm metabolism oxidative burst activity is concerned, it was observed that bulls, in which the burst activity was higher, resulted in better results of in vitro fertilization and on the further embryo production. The correlation between burst activity and fertilization rate and further embryo development to blastocysts was, respectively, $95.6 \%$ and $87.8 \%(P \leq .01)$. This study allows concluding that there is a positive correlation between bull sperm $\mathrm{H}_{2} \mathrm{O}_{2}$ production and their ability to fertilize bovine oocytes as well as their development to the blastocyst stage: sperm with high $\mathrm{H}_{2} \mathrm{O}_{2}$ production have high metabolic activities and consequently higher fertilizing capacities.

\section{Introduction}

Several techniques have been employed to determine the semen ability to fertilize oocytes, as it is generally accepted that there is a connection between the fertility of semen and its measurable properties. Motility and gross morphology, estimated by light microscopy, are by now the most used parameters for semen quality assessment, especially in artificial insemination laboratories. Due to the simplicity of the evaluation technique, motility is probably the most often used as criterion for routine semen evaluation. Motility may be divided in quantitative motility (percentage of sperm cells with a progressive motility) and qualitative motility. The latter involves several different parameters, some of which are the speed of the sperm moving, altitude of head displacement and movement pattern (circular versus linear movement, total distance versus progression, etc.). The accuracy in terms of repeatability of this test is, however, low, and dependent on the ability of the operator [1]. Probably for this reason, reports on the relationship between subjectively assessed sperm motility and fertility is inconsistent $[2,3]$. In fact, very few single sperm viability parameters show a significant relation with the fertility of the assayed frozenthawed semen sample, especially if it lies within accepted ranges of normality [4]. Therefore, there are no relation between in vitro tests as sperm capacitation, binding to the zona pellucida (ZP), acrosome reaction, oocyte fertilization (IVF), and in vitro embryo culture and the pregnancy rates after artificial insemination [5].

Computer assisted semen analysis (CASA) is an objective method that gives extensive information about the kinetic property of the ejaculate based on measurements of the individual sperm cells. Using CASA, motility, and movement characteristics of spermatozoa have been correlated to in 
vivo fertility [6-8]. Nevertheless, the major problem with this methodology is represented by the very high cost of the instruments that suggests its use only in laboratories performing high number of tests.

Concerning sperm metabolism, abundant literature has been published on the metabolic behaviour of spermatozoa of several species. In previous studies, Chaveiro et al. [9] described a flow cytometric method to evaluate apoptosis of frozen/thawed bovine sperm, which is directly related to the embryos development after in vitro fertilization [10]. Although hydrogen peroxide $\left(\mathrm{H}_{2} \mathrm{O}_{2}\right)$ is the major reactive oxygen species (ROS) produced by sperm [11], no study has been published relating the burst activity of sperm and their ability to fertilize. Spermatozoa, like all cells living under aerobic conditions, constantly face the oxygen paradox: oxygen is required for life, but the oxidative metabolism of biological molecules can be toxic due to the formation of highly reactive oxygen species that can modify cell functions and their viability. Particularly in sperm, high concentration of $\mathrm{H}_{2} \mathrm{O}_{2}$ is known to induce nuclear DNA fragmentation and lipid peroxidation resulting in cell death [12].

The most widely adapted dye to measure hydrogen peroxide is $2^{\prime} 7^{\prime}$ dichlorofluorecein diacetate (DCFH-DA). As a stable nonfluorescent compound, it diffuses into the cell by passive membrane diffusion. Once inside the cell, the dye is deacetylated by nonspecific esterases becoming a polar trapped polar $2^{\prime}, 7^{\prime}$-dichlorofluorescein (DCFH). Intracellular oxidation of DCFH during the metabolic burst, results in the formation of high green fluorescent $2^{\prime}, 7^{\prime}$ dichlorofluorescin which can further be measured by flow cytometry. The amount of formed DCF is proportional to the cellular oxidant production.

Therefore, the aim of the present study is to establish the relationship between the burst activity of bovine frozen/thawed sperm cells and its ability to fertilize in vitro.

\section{Materials and Methods}

Unless stated otherwise, all chemicals and media were purchased Sigma Chemical Company (St. Louis, USA) except FSH-LH that was obtained from Stimufol (Belgium).

2.1. Oocyte collection and In Vitro Maturation. Bovine ovaries were collected from a local slaughterhouse and transported to the laboratory in phosphate buffered saline (PBS) at $30-37^{\circ} \mathrm{C}$ within 2 hours. Cumulus-oocyte complexes (COCs) were aspirated from 2 to $6-\mathrm{mm}$ follicles with an $18 \mathrm{G}$ needle. The follicular fluid was deposited in a collecting tube filled with M199 buffered with $25 \mathrm{mM}$ Hepes supplemented with $10 \%$ oestrus cow serum, $5 \mu \mathrm{g} / \mathrm{mL}$ of gentamycin, $50 \mu \mathrm{g} / \mathrm{mL}$ of streptomycin, and $20 \mu \mathrm{g} / \mathrm{mL}$ of nystatin. After aspiration, only oocytes presenting a morphological appearance with multiple layers of unexpanded cumulus cells and evenly granulated and homogenous ooplasm, were recovered and selected for in vitro maturation (IVM). Selected COCs were first washed and then matured in M199 medium supplemented with $15 \%$ oestrus cow serum, $10 \mathrm{mg} / \mathrm{mL}$ of FSH-LH and $1 \mathrm{mg} / \mathrm{mL}$ of oestradiol- $17 \beta$. The maturation droplets (50 COC's in $500 \mu \mathrm{L}$ ) were incubated under mineral oil in an atmosphere of $5 \% \mathrm{CO}_{2}$ air at $39^{\circ} \mathrm{C}$ for 24 hours.

2.2. Semen Preparation and In Vitro Fertilization. Three straws ( $0.25 \mathrm{~mL}$ French straws) per bull of different ejaculates of a total of 8 bulls were thawed for 30 seconds in a water bath $\left(37^{\circ} \mathrm{C}\right)$. Afterwords, thawed straw content was split in two identical parts. One was used for in vitro fertilization, while the other one was used for flow cytometry to evaluate sperm viability and the sperm oxidative burst activity. Viable and mobile spermatozoa were separated by swim-up procedure as described by [9] remaining on the surface of the aliquot. Around $220 \mu \mathrm{L}$ of semen was kept under $1 \mathrm{~mL}$ of sperm-TALP medium in a sterile cryotube. This modified lactate-tyrode solution (TALP) contained $6 \mathrm{mg} / \mathrm{mL}$ of Bovine Albumin Serum (BSA) and $1.0 \mathrm{mM}$ of $\mathrm{Na}$-pyruvate $(\mathrm{pH}=7.2)$ was equilibrated in an atmosphere of $5 \% \mathrm{CO}_{2}$ air at $39^{\circ} \mathrm{C}$ for up to 45 minutes. While waiting this time to pass, the COC's were washed three times in fertilization medium before being transfereed to the 4-well plate. Each fertilization drop contained a TALP modified solution ( $\mathrm{pH}=7.6$ ) with $6 \mathrm{mg} / \mathrm{mL}$ of BAS-FAF (BAS-Fatty Acid Free) and $0.2 \mathrm{mM}$ of Na-pyruvate suplemented with $10 \mu \mathrm{g} / \mathrm{mL}$ of heparin, $20 \mu \mathrm{M}$ of D-penicillamine, $10 \mu \mathrm{M}$ of Hipotaurine, and $1 \mu \mathrm{M}$ of epinephrine. Ten $\mu \mathrm{L}$ of semen was taken before the swim-up procedure for evaluation of viability using DAPI $(3 \mu \mathrm{g} / \mathrm{mL})$ as a fluorescent dye (excitation 370, emission $470 \mathrm{~nm}$ ) for 5 minutes in the dark under a fluorescence microscope. After swim-up, the supernatant was centrifuged for 10 minutes $(200 \mathrm{xg})$ and the final pellet was homogenized to estimate the spermatozoa concentration on a hemocytometer. The insemination was performed by adding a final concentration of $1 \times 10^{6} \mathrm{spz} / \mathrm{mL}$ into fertilization drop included 25 to 30 oocytes $/ 500 \mu \mathrm{l}$ per drop. The incubation conditions were $5 \% \mathrm{CO}_{2}$ at $38.5^{\circ} \mathrm{C}$ in humidified air in a period of 18 hours of coincubation of spermatozoa and oocytes.

2.3. In Vitro Culture. After IVF, surrounding cumulus cells, spermatozoa, and cellular debris of presumptive zygotes were denuded by vortexing in fertilization medium for 2 minutes and subsequently washed before being transferred to the in vitro culture (IVC) drops (20-30 embryos/50 $\mu \mathrm{l}$ per drop). Embryos were allocated and cocultured with granulosa cells monolayer in B2 upgraded INRA medium (CCD Laboratory, Paris, France), supplemented with 10\% oestrus cow serum under mineral oil at $39^{\circ} \mathrm{C}$ in $5 \% \mathrm{CO}_{2}$. Cleavage and development of embryos to the blastocyst stage were assessed at 48 and 216 hours postinsemination (Day 0 defined as the day of fertilization), respectively.

2.4. Measurement of Sperm Respiratory Burst Activity. Per bull each ejaculated, the measurement was divided in six propylene flasks containing $500 \mu \mathrm{l} \mathrm{PBS}\left(37^{\circ} \mathrm{C}\right)$ to a final concentration of $1 \times 10^{6} \mathrm{spz} / \mathrm{mL}$, in duplicate. To observe the production of $\mathrm{H}_{2} \mathrm{O}_{2}$ in sperm cells, a method was used based on the combination of $2^{\prime}, 7^{\prime}$-dichlorofluoresceindiacetate (DCFH-DA) and phorbol myristate acetate (PMA), 
as previously described by Moreira da Silva et al. [13]. Briefly, the samples were incubated with DCFH-DA prepared in dimethyl sulfoxide (DMSO) to yield a final concentration of $5 \mu \mathrm{M}$, for 15 minutes at $37^{\circ} \mathrm{C}$, in a horizontal shaking water bath in the dark. Then, $0.5 \mu \mathrm{M}$ of phorbol myristate acetate (PMA) was added to activate the cellular metabolism of spermatozoa, and then incubated for other 15 minutes. The relative concentrations of $\mathrm{H}_{2} \mathrm{O}_{2}$ produced by the sperm cells were measured by a FacsCalibur flow cytometer (Becton Dickinson, San Jose, Calif, USA) after excitation at $469 \mathrm{~nm}$ and emission of green fluorescence at $541 \mathrm{~nm}$ through a $15 \mathrm{~mW}$ argon ion laser and data analyzed by the Cellquest software (Becton Dickinson, San Jose, Calif, USA). The mean fluorescence intensity of the analyzed sperm cells $(n=15000)$ was determined after gating the cell population by forward and side light scatter signals (FSC and SSC).

2.5. Statistical Analysis. Data obtained from oxidative product formation assays were analyzed by using analysis of variance (ANOVA) and the student's $t$-test was performed for all analyzes considering a $P$ value of $\leq .05$ for statistical significance.

\section{Results}

In this study, the relation between sperm $\mathrm{H}_{2} \mathrm{O}_{2}$ production and its ability to produce bovine embryos has been evaluated. Concerning sperm evaluation, the thawed sperm motility was on average $73 \%$ and no statistical difference was found among different bulls, as evaluated by the phase contrast microscopy. As expected, high correlation was obtained between sperm viability assessed by flow cytometry and microscopy $(r=0.95 ; P \leq .01)$.

There were no significant differences among bulls for viability and motility. Significant differences were found for the ability to produce IVF embryos as well for the cellular metabolism evaluated by the oxidative burst activity. Concerning the sperm oxidative burst activity, statistical differences were observed among bulls $(P \leq .001)$.

Our results showed some sperm bulls represented very low fluorescence intensity (25.7) while the others had very high burst activity (90.1) (Figure 1).

The maximum and the minimum cleavage rates were $86.0 \%$ and $49.5 \%$, while the maximum and minimum of blastocyst rates were $33.3 \%$ and $10.2 \%$. No statistical correlation was observed between the fertilization rate and the capacity of these zygotes to develop to blastocysts. Nevertheless, a high correlation was obtained between sperm $\mathrm{H}_{2} \mathrm{O}_{2}$ production and cleavage rate $(r=0.92 \%, P<.01)$ as well as the blastocysts rate $(r=0.80 \%, P \leq .05)$.

Two bulls had two sperm populations. Some sperm cells, after activation, represented high oxidative capacity of the DCFH, producing high fluorescence intensity of DCF, while the other sperm produced much low amount of DCF (Figure 2).

\section{Discussion}

Cells in general have mechanisms to produce reactive oxygen species (ROS) for physiological purposes, which can be produced by intracellular oxidases and peroxidases (e.g., xanthine oxidase), by leakage of electrons from the electron transport chain, regarded as toxic by products of other metabolic processes $[14,15]$. Another potential source of ROS production is reduction of oxygen by plasma membrane redox systems designed to transport reducing power across the cell plasma membrane [16]. In sperm, the mechanism of ROS is still unclear. It is known that freeze/thawing procedures of sperm provoke the ROS generation [17] by two different ways: (1) as a result of the NADPH-oxidase system at the level of the sperm plasma membrane, and (2) as a result of the NADPH-dependent oxidoreductase (diphorase) at the level of mitochondria $[18,19]$.

The first report that ROS could have harmful effects on sperm was published over 60 years ago [20]. A similar study performed by Aitken and Clarkson [21] in human sperm, but using the chemiluminescent probe, luminol, showed what it is now generally accepted: ROS production in sperm suspensions, lipid peroxidation and DNA oxidation were associated with poor sperm cells function and sub- or infertility. However, it is commonly accepted that cellular damage or death may only occur, if spermatozoa are in extreme oxidative conditions, or the antioxidant protective mechanisms of sperm cells are compromised. Oxidative stress (it is an imbalance between antioxidants and superoxide anions and hydrogen peroxide) is the result of an uncontrolled and excessive production of ROS, when it overwhelms the limited antioxidant defenses in semen. The excessive generation of ROS by the spermatozoa may result in peroxidation of polyunsaturated fatty acids (PUFs) of the plasma membrane [22]. As a result, the fluidity of the sperm membrane assured by the complex network of PUFs is compromised by the ROS and inhibits proper membrane fusion with the oocytes [21]. Hydrogen peroxide, in contrast to $\mathrm{O}_{2}^{-}$, is more stable and readily crosses the plasma membrane [14]. Several authors established that ROS can function as signaling molecules and evidence is emerging that sperm may generate low and controlled concentrations of ROS, specifically hydrogen peroxide $\left(\mathrm{H}_{2} \mathrm{O}_{2}\right)$, which is produced by spontaneous or enzymatic dismutation of $\mathrm{O}_{2}^{-}$. The hydrogen peroxide act to mediate the processes of capacitation (human: [23]; hamster: [24]), hyperactivation, and acrosome reaction (bovine: [25]; human: [26]) crucial to the acquisition of fertilizing ability [27].

In the present study, it was used the "swim-up" procedure, but as it has been reported, this method is associated with repeatedly centrifugation which increases ROS production $[28,29]$. Besides it may lead to cellular damage, by the fact that, when the sperm cells and seminal plasma are washed, much of the varieties of antioxidant protections are lost [30]. The oxidative metabolic burst activity of cells can be measured by assays which measure oxygen consumption, hexose monophosphate shunt activity, chemiluminescence, or generation of reactive oxygen derivates [31]. One accepted 


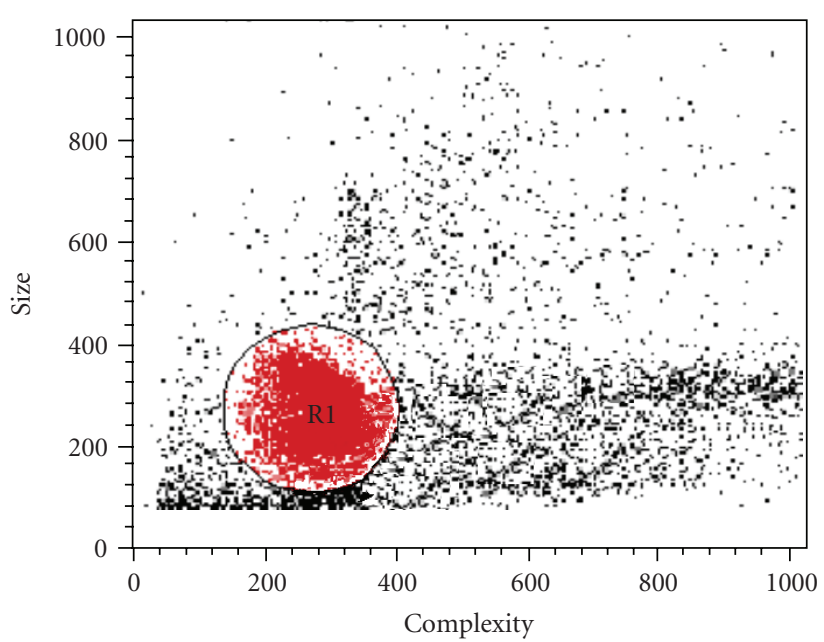

(a)

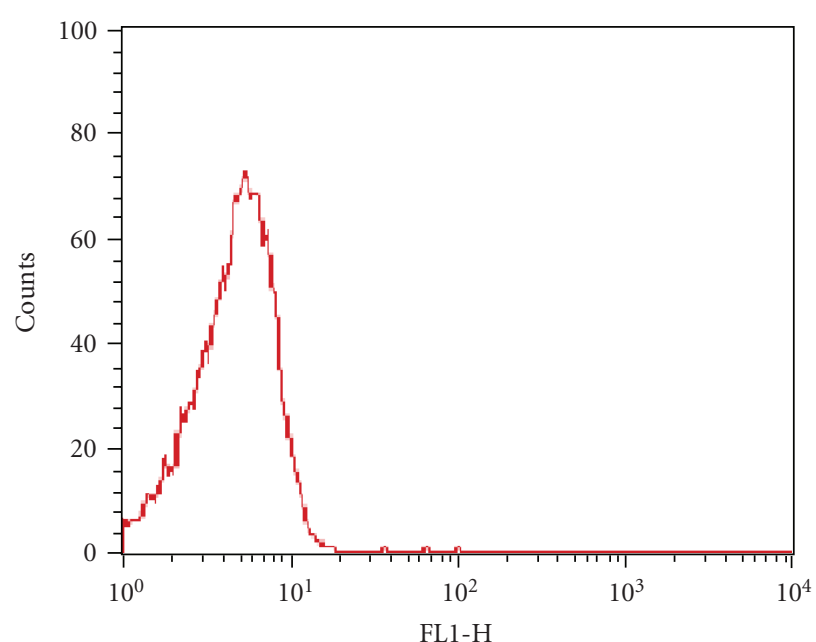

(b)

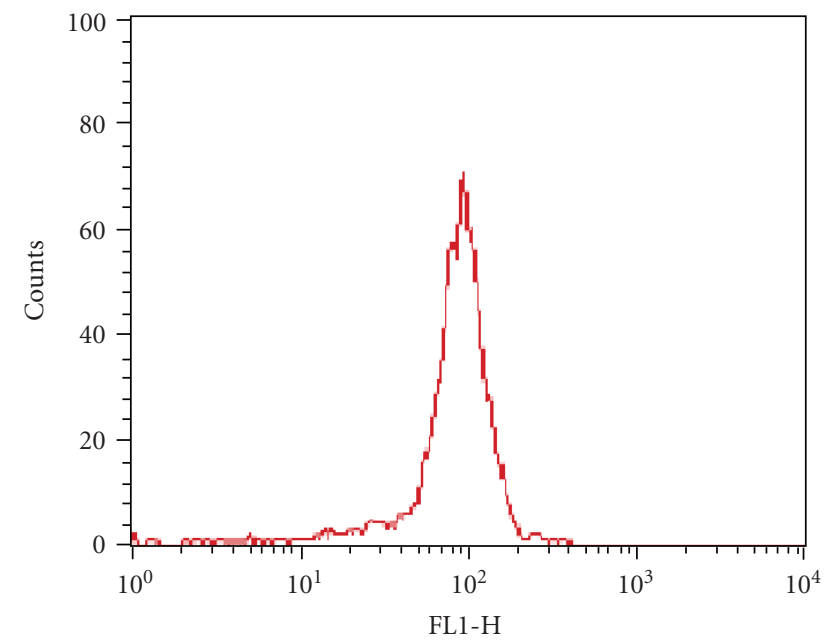

(c)

FIGURE 1: Typical results generated by flow cytometry evaluating the sperm burst activity, indirectly by the fluorescence of DCF. In (a) each dot represents a single cell; its position indicates its forward scatter (FSC) intensity value (cell size), and its side scatter (SSC) intensity value (cell granularity). The region indicated as R1 represents the population of the analyzed sperm cells. Both histograms indicate the number of sperm cells (counts) representing green fluorescence (FL1-H): (b) represents the bull with lowest sperm burst activity while (c) represents the bull with highest sperm burst activity.

technique for measuring oxidative metabolites is the quantification of hydrogen peroxide [32] by flow cytometry. In fact, the use of flow cytometry for sperm analysis is an attempt to address the long-standing problem of the subjective nature of the manual methods commonly used for semen analysis. This technique is more accurate, particularly more discriminative, allows for automatic, therefore rapid analysis of high number of cells in few seconds, and provides less subjective and statistically more reliable results than microscopic examination. In flow cytometry, the forward and sideways light scattering properties are considered to reflect cell size and the internal structure of cells, allowing separating the population we want to work on.

In this study, it was investigated the relation between the burst activity of bovine frozen/thawed sperm cells and their ability to fertilize in vitro bovine oocytes. To observe the production of $\mathrm{H}_{2} \mathrm{O}_{2}$ in sperm cells, a method was used based on the combination of $2^{\prime}, 7^{\prime}$-dichlorofluoresceindiacetate (DCFH-DA) and phorbol myristate acetate (PMA). It has been established that $\mathrm{H}_{2} \mathrm{O}_{2}$ is the main reactive oxygen species responsible for oxidative damage to the spermatozoon [22]. It is believed that either abnormal sperm, characterized by retention of excess residual cytoplasm as a result of defective spermatogenesis, or contaminating leukocytes in unpurified sperm suspensions are also sources for production of ROS [33]. Nevertheless, as afore described the use of flow cytometry allows to separate leukocytes or other debris from the sperm cells. DCFH-DA probe is not only oxidized by hydrogen peroxide but also its done by derived oxidants, other peroxides and indirectly by the superoxide anion when hydrogen peroxide is generated, thus providing a useful test to evaluate ROS production [34]. DCFH oxidation 


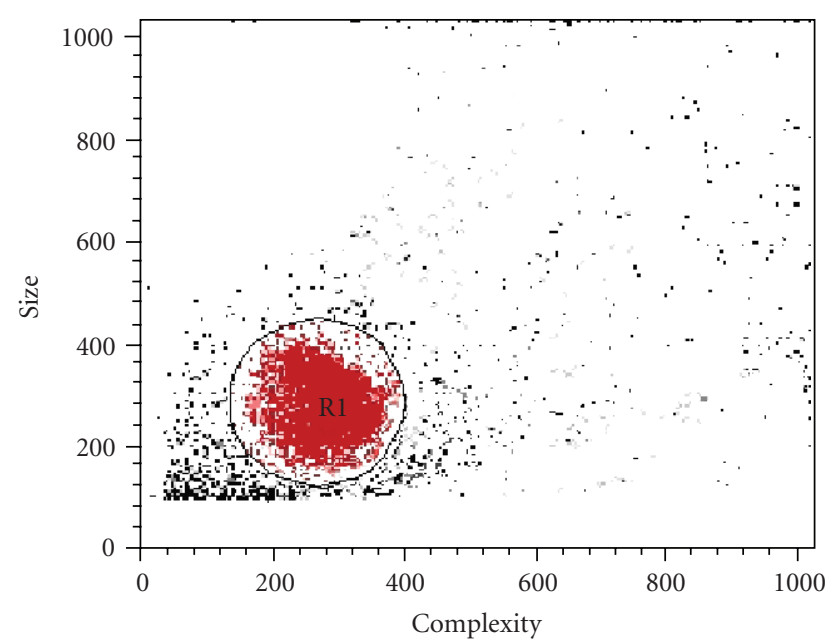

(a)

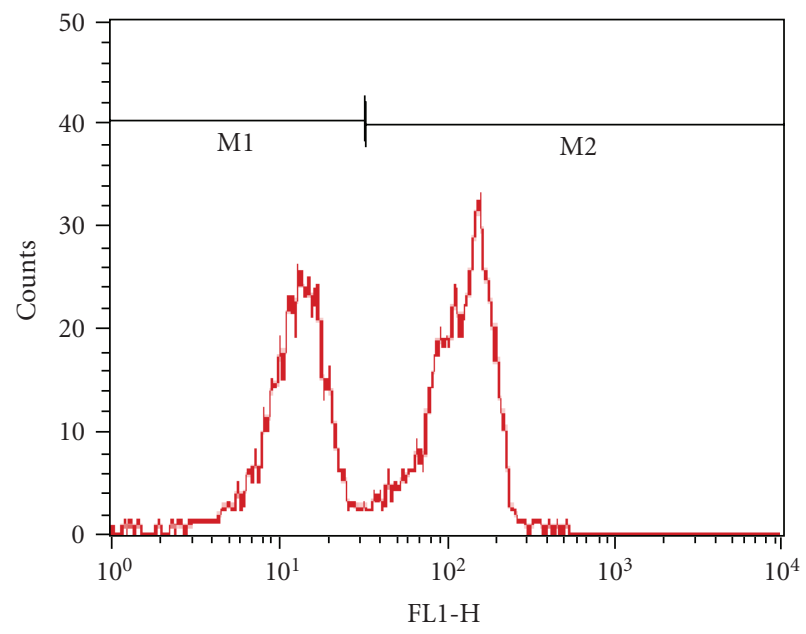

(b)

FIGURE 2: Typical results of sperm's oxidative burst with two distinct populations in the same straw. Besides as evaluated by means of FSC and SS the sperm population seem to be homogeneous (a), two distinct populations of burst activity are identified (b). One with low (M1) and the other with high fluorescence intensity (M2).

increases in a graduated fashion as a function of stimulus concentration. When the stable nonfluorescent compound (DCFH-DA) diffuses into the cell by passive membrane diffusion, it becomes a polar trapped DCFH compound cleaved by nonspecific intracellular estereases. The DCFH is nonfluorescent, but is rapidly oxidized to a highly green fluorescent dichlorofluorescein (DCF) by hydrogen peroxide. DCF formation reflects the oxidative burst of sperm cells, but its production depends on two enzyme systems, the esterease activity (during loading) and the hydrogen peroxide activity (i.e., upon stimulation). However minor burst stimulation generates only barely measurable DCF signals.

In previous flow cytometric studies, neutrophils or heterophils from mammalian species have produced a graded response in oxidative product formation relative to increasing concentrations of phorbol myristate acetate (PMA)
$[13,35]$. This was also exhibited by bovine spermatozoa, where the mean oxidative product formation increased after stimulation with PMA.

\section{Conclusion}

This study deals with the relations between bovine sperm $\mathrm{H}_{2} \mathrm{O}_{2}$ production and embryo production (cleavage rates and blastocyst formation). Our results showed a positive correlation between sperm $\mathrm{H}_{2} \mathrm{O}_{2}$ production and cleavage rate $(r=0.92)$ and between sperm $\mathrm{H}_{2} \mathrm{O}_{2}$ production and blastocysts formation $(r=0.8)$. Generation of $\mathrm{H}_{2} \mathrm{O}_{2}$ is not merely a means of discarding toxic waste products, but instead of that it plays a significant role in sperm metabolism. If sperm cells generate low amounts of ROS, it can be the result of a low metabolic activity, with low ability to fertilize. One can suggest that measurement of intracellular $\mathrm{H}_{2} \mathrm{O}_{2}$ concentration can thus be a valuable tool to predict the sperm fertilization capacity: sperm cells with high $\mathrm{H}_{2} \mathrm{O}_{2}$ concentration have high metabolic activities and consequently higher fertilizing capacities.

\section{Acknowledgments}

The first author is supported by the Regional Foundation for Science and Technology of the Azores Government.

\section{References}

[1] R. S. Jeyendran and E. F. Graham, "An evaluation of cryoprotective compounds on bovine spermatozoa," Cryobiology, vol. 17, no. 5, pp. 458-464, 1980.

[2] A. Januskauskas, M. G. Håård, M. C. Håård, L. Söderquist, N. Lundeheim, and H. Rodriguez-Martinez, "Estimation of sperm viability in frozen-thawed semen from Swedish A.I. bulls," Zentralblatt für Veterinärmedizin. Reihe A, vol. 43, no. 5, pp. 281-287, 1996.

[3] H. Kjaestad, E. Ropstad, and K. A. Berg, "Evaluation of spermatological parameters used to predict the fertility of frozen bull semen," Acta Veterinaria Scandinavica, vol. 34, no. 3, pp. 299-303, 1993.

[4] R. P. Amann and R. H. Hammerstedt, "Andrology lab corner: detection of differences in fertility," Journal of Andrology, vol. 23, no. 3, pp. 317-325, 2002.

[5] H. Rodríguez-Martínez, "Laboratory semen assessment and prediction of fertility: still utopia?" Reproduction in Domestic Animals, vol. 38, no. 4, pp. 312-318, 2003.

[6] P. R. Budworth, R. P. Amann, and P. L. Chapman, "Relationships between computerized measurements of motion of frozen-thawed bull spermatozoa and fertility," Journal of Andrology, vol. 9, no. 1, pp. 41-54, 1988.

[7] C. Holt, W. V. Holt, H. D. M. Moore, H. C. B. Reed, and R. M. Curnock, "Objectivily measured boar sperm motility parameters correlate with the outcomes of on-farm inseminations: results of two fertility trials," Journal of Andrology, vol. 18, no. 3, pp. 312-323, 1997.

[8] B. R. Zhang, B. Larsson, N. Lundeheim, and H. RodriguezMartinez, "Sperm characteristics and zona pellucida binding in relation to field fertility of frozen-thawed semen from dairy AI bulls," International Journal of Andrology, vol. 21, no. 4, pp. 207-216, 1998. 
[9] A. Chaveiro, P. Santos, and F. M. da Silva, "Assessment of sperm apoptosis in cryopreserved bull semen after swim-up treatment: a flow cytometric study," Reproduction in Domestic Animals, vol. 42, no. 1, pp. 17-21, 2007.

[10] G. Antunes, A. Chaveiro, P. Santos, A. Marques, H. S. Jin, and F. Moreira da Silva, "Influence of apoptosis in bovine embryo's development," Reproduction in Domestic Animals. Epub ahead of print.

[11] F.-J. Liu, G. P. Cobb, T. A. Anderson, Q.-Q. Cheng, and C. W. Theodorakis, "Uptake, accumulation and depuration of sodium perchlorate and sodium arsenate in zebrafish (Danio rerio)," Chemosphere, vol. 65, no. 10, pp. 1679-1689, 2006.

[12] R. Kasimanickam, K. D. Pelzer, V. Kasimanickam, W. S. Swecker, and C. D. Thatcher, "Association of classical semen parameters, sperm DNA fragmentation index, lipid peroxidation and antioxidant enzymatic activity of semen in ramlambs," Theriogenology, vol. 65, no. 7, pp. 1407-1421, 2006.

[13] F. Moreira da Silva, Studies on reproduction and immunity: effect of the reproductive hotmones on the oxidative burst activity of bovine neutrophils, Ph.D. thesis, University of Gent, Gent, Belgium, 1997.

[14] B. Halliwell, "Reactive oxygen species in living systems: source, biochemistry, and role in human disease," American Journal of Medicine, vol. 91, no. 3, pp. 14S-22S, 1991.

[15] S. Raha and B. H. Robinson, "Mitochondria, oxygen free radicals, disease and ageing," Trends in Biochemical Sciences, vol. 25, no. 10, pp. 502-508, 2000.

[16] A. D. N. J. de Grey, "A hypothesis for the minimal overall structure of the mammalian plasma membrane redox system," Protoplasma, vol. 221, no. 1-2, pp. 3-9, 2003.

[17] J. F. H. M. Brouwers and B. M. Gadella, "In situ detection and localization of lipid peroxidation in individual bovine sperm cells," Free Radical Biology and Medicine, vol. 35, no. 11, pp. 1382-1391, 2003.

[18] B. A. Ball, A. T. Vo, and J. Baumber, "Generation of reactive oxygen species by equine spermatozoa," American Journal of Veterinary Research, vol. 62, no. 4, pp. 508-515, 2001.

[19] B. Bánfi, G. Molnár, A. Maturana, et al., "A Ca ${ }^{2+}$-activated NADPH oxidase in testis, spleen, and lymph nodes," The Journal of Biological Chemistry, vol. 276, no. 40, pp. 37594 37601, 2001.

[20] J. McLeod, "The role of oxygen in the metabolism and motility of human spermatozoa," American Journal of Physiology, vol. 138, pp. 512-518, 1943.

[21] R. J. Aitken and J. S. Clarkson, "Cellular basis of defective sperm function and its association with the genesis of reactive oxygen species by human spermatozoa," Journal of Reproduction and Fertility, vol. 81, no. 2, pp. 459-469, 1987.

[22] R. J. Aitken, D. Buckingham, and D. Harkiss, "Use of a xanthine oxidase free radical generating system to investigate the cytotoxic effects of reactive oxygen species on human spermatozoa," Journal of Reproduction and Fertility, vol. 97, no. 2, pp. 441-450, 1993.

[23] J. F. Griveau, P. Renard, and D. Le Lannou, "An in vitro promoting role for hydrogen peroxide in human sperm capacitation," International Journal of Andrology, vol. 17, no. 6, pp. 300-307, 1994.

[24] I. Bize, G. Santander, P. Cabello, D. Driscoll, and C. Sharpe, "Hydrogen peroxide is involved in hamster sperm capacitation in vitro," Biology of Reproduction, vol. 44, no. 3, pp. 398-403, 1991.

[25] C. O’Flaherty, N. Beorlegui, and M. Beconi, "Role of superoxide anion and hydrogen peroxide in acrosome reaction of bovine spermatozoa," in Andrology in the 21st Century, B.
Robaire, H. Chemes, and C. Morales, Eds., pp. 103-108, 2001.

[26] E. de Lamirande, H. Jiang, A. Zini, H. Kodama, and C. Gagnon, "Reactive oxygen species and sperm physiology," Reviews of Reproduction, vol. 2, no. 1, pp. 48-54, 1997.

[27] P. Leclerc, E. de Lamirande, and C. Gagnon, "Regulation of protein-tyrosine phosphorylation and human sperm capacitation by reactive oxygen derivatives," Free Radical Biology and Medicine, vol. 22, no. 4, pp. 643-656, 1997.

[28] A. Agarwal and T. M. Said, "Oxidative stress, DNA damage and apoptosis in male infertility: a clinical approach," BJU International, vol. 95, no. 4, pp. 503-507, 2005.

[29] M. Shekarriz, A. J. Thomas Jr., and A. Agarwal, "Incidence and level of seminal reactive oxygen species in normal men," Urology, vol. 45, no. 1, pp. 103-107, 1995.

[30] S. E. M. Lewis, E. S. L. Sterling, I. S. Young, and W. Thompson, "Comparison of individual antioxidants of sperm and seminal plasma in fertile and infertile men," Fertility and Sterility, vol. 67, no. 1, pp. 142-147, 1997.

[31] S. Klebanoff and R. Clark, "The metabolic burst," in The Neutrophil: Function and Clinical Disorders, S. J. Klebanoff and R. A. Clark, Eds., pp. 283-408, Elsevier/North-Holland Biomedical Press, Amsterdam, The Netherlands, 1978.

[32] D. A. Bass, J. W. Parce, L. R. Dechatelet, P. Szejda, M. C. Seeds, and M. Thomas, "Flow cytometric studies of oxidative product formation by neutrophils: a graded response to membrane stimulation," Journal of Immunology, vol. 130, no. 4, pp. 1910-1917, 1983.

[33] K. Whittington and W. C. L. Ford, "Relative contribution of leukocytes and of spermatozoa so reactive oxygen species production in human sperm suspensions," International Journal of Andrology, vol. 22, no. 4, pp. 229-235, 1999.

[34] C. P. LeBel, H. Ischiropoulos, and S. C. Bondy, "Evaluation of the probe $2^{\prime}, 7^{\prime}$-dichlorofluorescin as an indicator of reactive oxygen species formation and oxidative stress," Chemical Research in Toxicology, vol. 5, no. 2, pp. 227-231, 1992.

[35] A. Chaveiro and F. Moreira da Silva, "Effect of oestrous cycle on the oxidative burst activity of blood polymorphonuclear leucocytes in cows," Reproduction in Domestic Animals. Epub ahead of print. 